\title{
Coil Design and Optimization of Inductive Power Transfer System for Tram
}

\author{
Liwei Zhang ${ }^{1}$, Fengyu Leng ${ }^{1 *}$, Sanmu Xiư', Wenmei Hao ${ }^{1}$ \\ 1 School of Electrical Engineering, Beijing Jiaotong University, No. 3 Shangyuancun, Haidian District, Beijing 100044, China \\ *Corresponding author, e-mail: 16121471@bjtu.edu.cn
}

Received: 12 November 2018, Accepted: 13 November 2018, Published online: 12 March 2020

\begin{abstract}
As a new type of urban transit vehicle, Non-catenary trams using inductive power transmission technology get rid of the traditional overhead catenary. In engineering applications, coils assembled on the different tram bodies have inevitable differences due to the restrictions on the production process and other factors. Research shows tiny differences in self-inductance always lead to system detuning so as to causes an extreme descent of the system power factor. From the perspective of hardware design, the paper analyzes the system architecture and coil configuration for the dynamic charging trams with considering cost, system reliability, etc. Then, for the problem of power factor reduction caused by the differences in the self-inductance of the secondary windings, the article establishes a mathematical model with the maximum power factor as the goal and system parameters as constraints. And a complete system parameters design method is proposed. Finally, the global design and optimization of tram's electromagnetic coupling mechanism parameters are performed using the group method. The simulation result indicates that the method can meet the requirements of system operation and has a higher tolerance to the self-inductance differences of the secondary coils.
\end{abstract}

\section{Keywords}

inductive power transfer, coil design and optimization, dynamic charging, high power

\section{Introduction}

As a new type of urban transit vehicle, the non-catenary trams use Inductive Power Transfer (IPT) technology for wireless power transmission, enabling the power supply system and the vehicle to transmit energy without physical contact. The IPT technology not only solves the disadvantages brought by the traditional contact power supply mode, such as easy loss, contact spark, carbon accumulation, difficult maintenance, high construction cost, but improves the safety, reliability and flexibility of vehicle operation (Li et al., 2015; Yang et al., 2010; Zhao et al., 2016). Applying IPT technology to urban transit vehicles must meet trams' power supply requirements include high-power and high-efficiency The coil in the IPT system serves as a carrier for energy transmission. And its laying mode and parameter design will have a direct impact on energy transfer power and efficiency. Therefore, research on coupled coils is particularly important (Budhia et al., 2011; Fan et al., 2018; Li et al., 2010).

At present, domestic and foreign scholars' research on coupled coils in IPT systems mainly includes: from the perspective of coil shape design, a DLDD type coil structure for electric vehicles is proposed in the research (Hu et al., 2013), which is analyzed and proved to have good offset tolerance. In the study of Xu et al. (2018), the magnetic circuit models of the coupling system of the primary and secondary coils are established respectively. Then, the magnetic core structure is optimized by deriving the approximate calculation formula of the primary inductances, secondary inductances and the coupling coefficient. In order to improve the power transmission capability of the coils, Sun et al. (2010) propose to optimize the mutual inductance parameter between the primary and secondary coils with the energy efficiency product index. Geng et al. (2017) and Su et al. (2016) increase the efficiency or output power of the system by increasing the number of parallels of the primary and secondary coils. It can be seen that most of the existing papers focus on a certain index of power or efficiency, or adopt different optimization processes and evaluation methods, optimize the performance parameters of a certain aspect 
of the coupled coils, and do not give the coils parameter design method of the system. Due to the important performance indexes such as power and efficiency often have opposite requirements on the parameters of the coil turns, mutual inductance, etc. Therefore, the parameters of the coupled coils need to be globally designed.

Taking non-catenary trains using IPT technology as the application background, the article takes into account the actual installation space, axle load, system reliability, cost etc. And analyzes the coil configuration method suitable for dynamic charging trams. Then, the specific design method of the parameters such as mutual inductance and number of turns of the coil is given. When the coil is manufactured, the accuracy of the self-inductance parameters of each coil is limited and there must be differences in each coil. The differences in self-inductance of the primary coils fixed on the ground can be adjusted by changing the switching frequency of the converter timely, while the differences in the secondary coils installed on the different carriage in high-speed motion are difficult to achieve realtime compensation. Therefore, this paper proposes a mathematical model with system parameters as constraints and maximum power factor as the goal for the power factor reduction caused by the self-inductance differences of the secondary coil. Finally, the global design and optimization method of trains' electromagnetic coupling mechanism parameters are performed using the group method. Simulation results demonstrate the effectiveness of the design optimization method. Moreover, the method can improve the tolerance of the system to the differences in the secondary coil self-inductance effectively.

\section{IPT system coil structure}

The coil shapes commonly used in IPT system include circular, rectangular, and combined coil (DD) (Cai et al., 2014). The circular coil has consistent direction but poor offset tolerance. The combined coil (DD) is complicated and costly. In contrast, the rectangular coil is easy to twine and ensure the uniformity of the air gap magnetic field. And the distortion at the corner of the coils can be reduced by setting a certain chamfer, which is suitable for the tram that has an offset only in a single direction. Therefore, this article uses the rectangular coil.

Fig. 1 is the actual structure of the non-catenary tram and the installation space of the coupled coils using IPT technology. Different from the way of static charging which lays the coil only at the platforms, dynamic charging can lay a certain length of the primary coils in the middle of the rails according to distance between stations, the stopping time

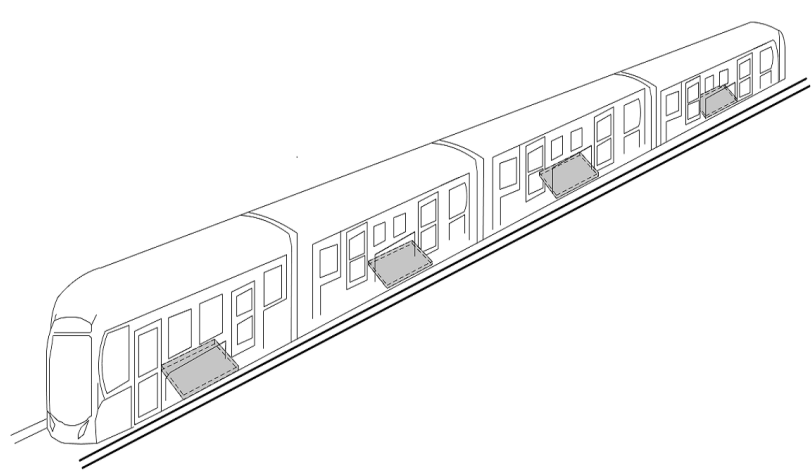

Fig. 1 Structure of the non-catenary tram and installation space of IPT system

and the vehicle energy consumption (Zhang et al., 2017). And the secondary coils are installed on the bottom of the trains. It is known that the mutual inductance of the primary and secondary coils can be expressed as:

$M=\frac{\varphi}{I}=\frac{B S}{I}$.

It can be known from Eq. (1) that the mutual inductance value $M$ between the coils is determined by the value of the magnetic induction intensity $B$ in the plane $S$ included in the coil, where the magnitude of the magnetic induction intensity $B$ and the current $I$ is directly or indirectly affected by the system input voltage level, the converter control strategy, and the like. Therefore, in the same situation, increasing the coil area can increase the mutual inductance value while saving the winding. In addition, the literature of $\mathrm{Hu}$ et al. (2013) mentions that increasing the coil area is beneficial to increase the coupling coefficient between the primary and secondary coils. Consequently, the secondary coil size is targeted to maximize the use of the assembly space provided by the vehicle, and the primary coil width is consistent with the secondary coil to improve coupling coefficient. Based on the above analysis, the paper proposes to adopt a coil configuration method in which the primary coils are long and the secondary coils are short as shown in Fig. 2.

Import the structure of Fig. 2 into simulation software Ansys Maxwell and we can get the magnetic induction intensity distribution of different distances above the primary coil as shown in Fig. 3 The analysis shows that the longer coil configuration of the primary side can keep the magnetic field in the middle of the coil stable, and the magnetic field fluctuation at the coil boundary becomes smaller as the distance increases. Therefore, the structure can reduce the magnetic field fluctuations at the boundary of the coils and is advantageous for the stable output of the system. 


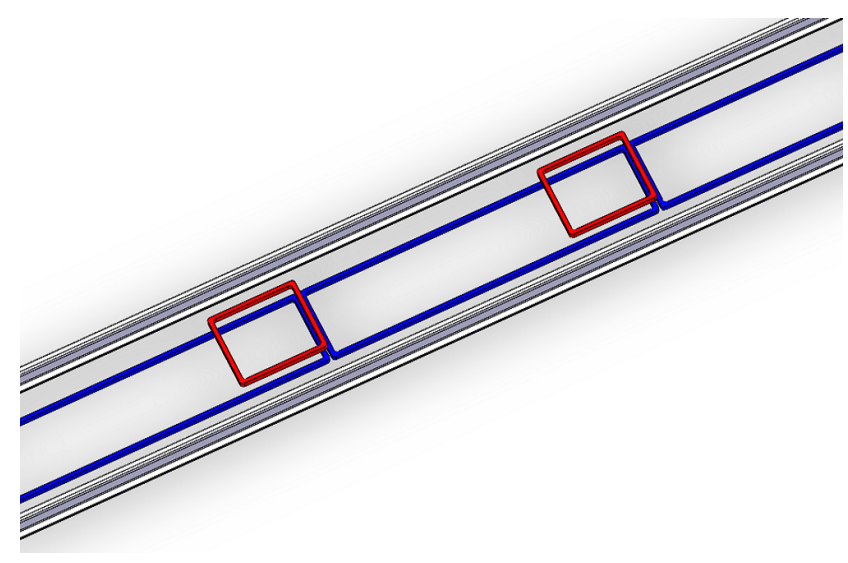

Fig. 2 Configuration of primary and secondary coils

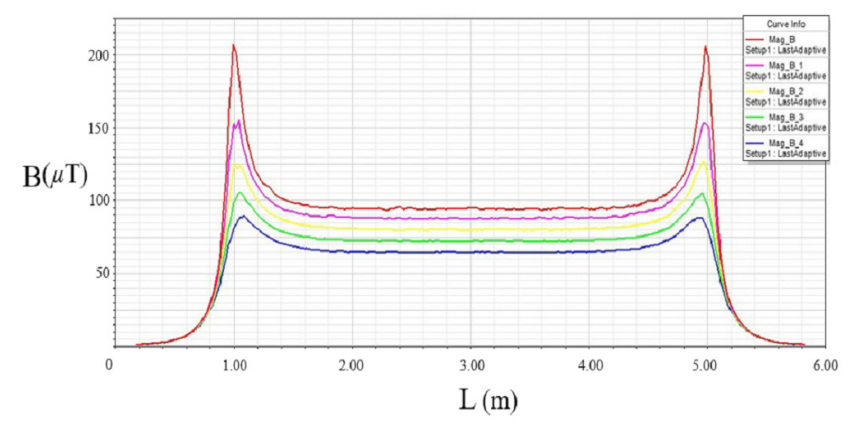

Fig. 3 Distribution of single-turn coil's magnetic induction

\section{IPT system coil parameters design}

In addition to the size and distance of the primary and secondary coils determined by the structure of the trams' body, the mutual inductance, self-inductance, resistance, and number of turns will have a direct impact on system performance. It will be analyzed below.

\subsection{IPT System Topology}

In the IPT system, the large air gap between the primary and secondary coils causes a large leakage inductance in the coupled coil. If no compensation is applied, the power transmission capability of the system will be greatly affected and the voltage and current stress experienced by the power device will be increased. To solve this problem, capacitors can be connected in series or in parallel on the primary and secondary coils. Common compensation methods can be divided into SeriesSeries (SS) compensation, Series-Parallel (SP) compensation, Parallel-Series (PS) compensation, and Parallel-toParallel (PP) compensation (Tan et al., 2010; Zheng, 2017). Among them, the series-series compensation has the characteristics of simple structure and constant output voltage. And its compensation capacitance value is independent of the mutual load and inductance between the original and secondary coils. The feature is suitable for the case where there is mutual inductance and load fluctuation during the movement of the tram. Therefore, the paper analyzes the IPT system based on the series-series compensation topology. The main circuit topology of the system is shown in Fig. 4.

Among them, $U_{d c}$ and $U_{o}$ are the DC bus voltage and the DC voltage output from the secondary side. $U_{1}$ is the effective value of the inverter output voltage, and $U_{2}$ is the effective value of the voltage before the secondary rectifier. $I_{1}$ and $I_{2}$ are current effective value flowing through the primary and secondary coils. $L_{1}, L_{2}, R_{1}$ and $R_{2}$ are the inductance and internal resistance of the primary and secondary coils, respectively. $C_{1}$ and $C_{2}$ are the primary and secondary compensation capacitors. $R_{l}$ is the load resistance. And $M$ is the mutual inductance between the primary and secondary coils. Fig. 4 is simplified to obtain the SS compensation topology simplification circuit shown in Fig. 5.

Then the system meets:

$\left\{\begin{array}{l}U_{1}=\frac{2 \sqrt{2} U_{d c}}{\pi} \\ U_{2}=\frac{2 \sqrt{2} U_{o}}{\pi} . \\ R_{e}=\frac{8}{\pi^{2}} R_{l}\end{array}\right.$

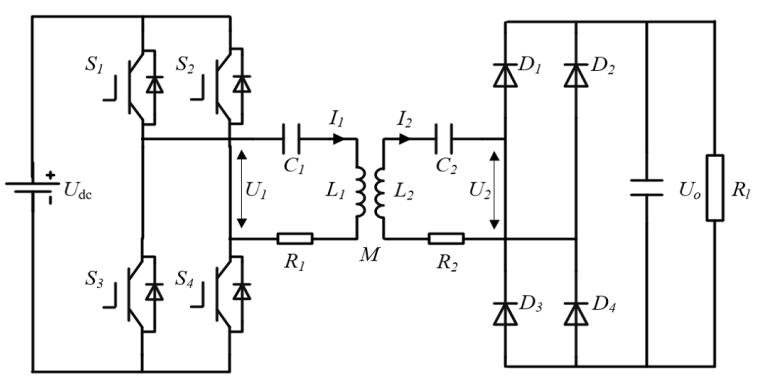

Fig. 4 Topology of series-series compensated IPT system

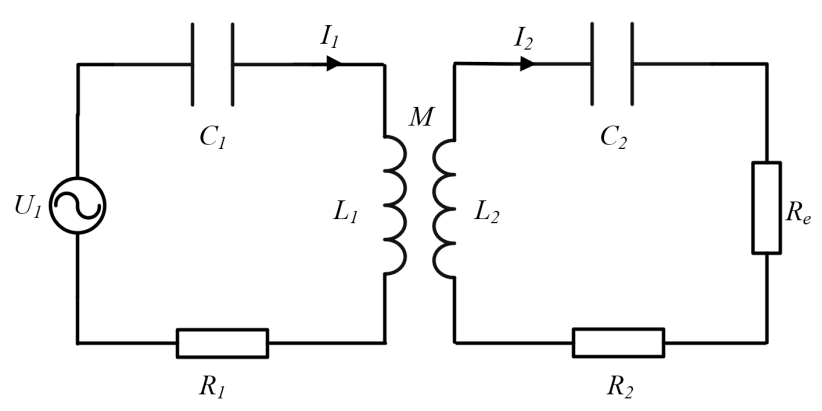

Fig. 5 Simplified series-series compensated IPT system 
When the primary and secondary coils resonate at the angular frequency $\omega_{0}$, namely:

$\omega_{0}^{2} L_{1} C_{1}=\omega_{0}^{2} L_{2} C_{2}=1$.

At this point, the output power and efficiency of the system are:

$$
\begin{aligned}
& P_{\text {out }}=\frac{R_{e} \omega_{0}^{2} M^{2} U_{1}^{2}}{\left[R_{1}\left(R_{e}+R_{2}\right)+\omega_{0}^{2} M^{2}\right]^{2}}, \\
& \eta=\frac{R_{e} \omega_{0}^{2} M^{2}}{\left(R_{e}+R_{2}\right)\left[\omega_{0}^{2} M^{2}+R_{1}\left(R_{e}+R_{2}\right)\right]} .
\end{aligned}
$$

It can be known from Eqs. (4) and (5) that the system output power and efficiency are directly affected by the coils' resistance and the mutual inductance between the primary and secondary coils. The mutual inductance and resistance are related to the self-inductance and the number of turns of the coils. Therefore, it is necessary to properly design the coils parameters to meet the system's requirements for output power and efficiency.

\subsection{Design algorithm of coil parameters}

\subsubsection{Mutual inductance parameter algorithm}

Mutual inductance is one of the important parameters in the design process of the coupled coil. Sun et al. (2010) and $\mathrm{Li}$ et al. (2010) aim at the maximum energy efficiency product and maximum output power respectively, and give the optimal mutual inductance calculation method. However, it can be seen from the calculation formula that to calculate the mutual inductance value must be under the condition that the number of turns of the primary and secondary coils and the resistance have been completely determined. In engineering applications, if you want to achieve the calculated optimal mutual inductance value, you can only change the air gap between the primary and secondary coils continuously. Therefore, the prior method is not suitable for the occasion where the primary and secondary coils' distance of the electromagnetic coupling mechanism cannot be adjusted at will. Considering that the power supply mode of urban vehicles is converted from DC distribution network power supply to induction power transmission and in order to achieve the minimum modification of the existing trams, the paper is compatible with the current power supply voltage level of the vehicle converter assembly. Thus the output voltage of the electromagnetic coupling mechanism is $U_{o}=U_{d c}=750 \mathrm{~V}$. Introducing the system voltage gain $G_{U}$, i.e.:
$G_{U}=\frac{U_{o}}{U_{d c}}$

The mutual inductance value $M$ between the original and secondary coils can be obtained as:

$M=\frac{R_{e}}{\omega_{0} G_{U}}$.

\subsubsection{Coil turn matching algorithm}

According to the Niemann formula, the self-inductance and the mutual inductance calculation formula of the rectangular coils are:

$L_{s}=\frac{\mu_{0}}{4 \pi}\left[\begin{array}{l}\frac{2 a b}{r\left(a+\sqrt{a^{2}+b^{2}}\right)}+b \ln \frac{2 a b}{r\left(a+\sqrt{a^{2}+b^{2}}\right)} \\ -2\left(a+b-\sqrt{a^{2}+b^{2}}\right)+\frac{a+b}{4}\end{array}\right]$,

$M_{s}=\frac{\mu_{0}}{4 \pi} \oint_{l_{1}} \oint_{l_{2}} \frac{d l_{1} d l_{2}}{R}$

where $a, b$, and $r$ are the length, width of the rectangular coil and the cross-section radius of the wire, respectively. $\mu_{0}$ is the magnetic permeability in the vacuum. Therefore, when the coil structure parameters are determined, the self-inductance values of the single-turn primary, secondary coils and the mutual inductance values between the single-turn primary and secondary coils can be calculated as $L_{10}, L_{20}, M_{0}$, respectively. If the number of turns and the self-inductance of the primary and secondary coils are $N_{1}, N_{2}, L_{1}$, and $L_{2}$, respectively, then:

$M=N_{1} N_{2} M_{0}$

$L_{1}=N_{1}^{2} L_{10}$

$L_{2}=N_{2}^{2} L_{20}$.

By combining Eqs. (8)-(10), the number of turns of the primary and secondary coils that meet the mutual inductance requirements can be calculated. Since the result of the number of turns is not unique, it is necessary to optimize the parameters of each group.

\section{Optimization of coil parameters based on differences of secondary self-inductance}

\subsection{Target of parameters optimization}

In practical applications, due to factors such as coil manufacturing process and structural deformation, the accuracy of each coil can only be maintained at $5 \%-10 \%$. And multiple 
original and secondary coils are difficult to achieve parameter consistency. However, the self-inductance deviation of the primary and secondary coils will directly affect the system resonance, which in turn affects the system ability to transmit power. For the primary coil fixed on the ground, the difference between the theoretical calculated value and the actual parameter can be adjusted by changing the switching frequency of the converter device, so that the primary coil $L_{1}$ and the primary compensation capacitor $C_{1}$ completely resonate at the frequency $\omega_{0}$. However, for each secondary coil installed on different carriages, when the train passes the charging section at an average speed of $70 \mathrm{~km} / \mathrm{h}$, the response speed of the detection control device is limited, and it is difficult to adjust the resonance frequency of the system timely. And ensure that it has the same resonant frequency as the primary coil. In the case where the primary coil is completely resonated and the secondary side coil has a self-inductance deviation, the primary and secondary coils' impedances $Z_{1}$ and $Z_{2}$ are expressed by Eqs. (13) and (14), respectively. Since the internal resistance of the high-frequency Litz wire used for winding the coil is small, the internal resistance of the coil is neglected.

$$
\begin{aligned}
& Z_{1}=j \omega_{0} L_{1}+\frac{1}{j \omega_{0} C_{1}}=0 \\
& Z_{2}=j \omega_{0} L_{2}+\frac{1}{j \omega_{0} C_{2}}+R_{e}
\end{aligned}
$$

At this point, the active power of the system can be expressed as:

$$
P_{\text {out }}=\frac{R_{e} U_{1}^{2}}{\omega_{0}^{2} M^{2}} \cdot \lambda=\frac{R_{e} U_{1}^{2}}{\omega_{0}^{2} M^{2}} \cdot \frac{R_{e}}{\sqrt{R_{e}^{2}+\left(\omega_{0} L_{2}+\frac{1}{\omega_{0} C_{2}}\right)^{2}}} .
$$

In the Eq. (15), $\lambda$ is the power factor of the secondary coil. It can be seen from Eq. (15) that the output power of the system is related to the power factor of the secondary coil. And the power factor of the secondary coil is affected by the self-inductance. When the system operating voltage is $U_{d c}=750 \mathrm{~V}$ and the working frequency is $f=30 \mathrm{kHz}$, the three sets of coil self-inductance and compensation capacitance parameter values satisfying the system requirements are selected as shown in Table 1 according to the coil parameters design algorithm described in Subsection 3.2. And Fig. 6 shows the relationship between the power factor and the self-inductance deviation of the secondary coil.

As shown in Fig. 6, when the theoretical calculation requires a secondary coil's self-inductance of $998 \mathrm{uH}$, but an inductance deviation of $10 \%$, that is, the actual inductance
Table 1 The self-inductance value of the coil and the value of the compensation capacitor

\begin{tabular}{lcccc}
\hline Parameters & $L_{1} / \mathrm{uH}$ & $C_{1} / \mathrm{nF}$ & $L_{2} / \mathrm{uH}$ & $C_{2} / \mathrm{nF}$ \\
\hline Curve 1 & 15.3 & 1840 & 998.0 & 28.2 \\
Curve 2 & 123.7 & 227.5 & 123.7 & 227.5 \\
Curve 3 & 998.0 & 28.2 & 15.3 & 1840 \\
\hline
\end{tabular}

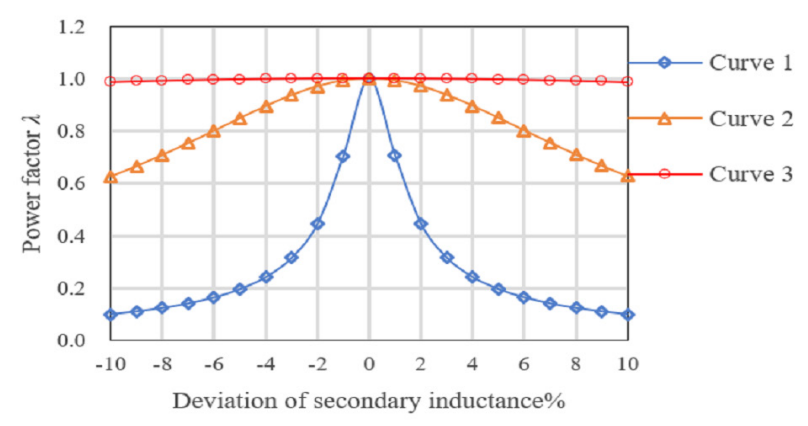

Fig. 6 Relationship between power factor and deviation of secondary inductance

value is $1097 \mathrm{uH}$, the system's power factor is only 0.099 . It will greatly affect system performance. Analysis of the set of data shows that the greater the self-inductance value of the secondary coil, the worse the tolerance of the system with the self-inductance deviation, which will lead to a sharp decrease in the system power factor. Based on the above analysis, the optimization goal of the paper is the maximum power factor in case of deviation.

\subsection{System constraints on coil parameters}

In addition to the coil's own parameters, other indicators in the system also have certain constraints on the coil parameters.

\subsubsection{Constraint of compensation capacitance}

According to the circuit theory, in the series resonant circuit, the quality factor $Q$ of the resonant circuit is defined as:

$$
Q=\frac{\omega_{0} L}{R}=\frac{1}{\omega_{0} C R}=\frac{1}{R} \sqrt{\frac{L}{C}} .
$$

When the coil is connected in series with the compensation capacitor, the voltage value on the compensation capacitor is $U_{c}=Q U_{s}$. Obviously, when $Q>1$, the voltage $U_{c}$ across the capacitor will have an overvoltage that is $Q$ times higher than the power supply voltage. Considering the withstand voltage of the compensation capacitor, we need to limit the self-inductance of the coil. Designed with 1.5 times voltage margin, the constraints of the compensation capacitor on the coil self-inductance are: 


$$
\begin{aligned}
& \mathrm{L}_{1} \leq \frac{2 \omega_{0} M^{2} U_{C}}{3 U_{1} R_{e}} \\
& \mathrm{~L}_{2} \leq \frac{2 R_{e} U_{C}}{3 \omega_{0} U_{2}} .
\end{aligned}
$$

\subsubsection{Constraints of output power and efficiency}

In order to ensure the normal operation of the tram, the system must meet the output power and efficiency requirements under the rated condition. It can be seen from Eqs. (4) and (5) that when calculating the output power and efficiency, the internal resistance of the primary and secondary coils must also be considered. The inductively coupled coil uses Litz wire whose equivalent AC impedance ESR can be expressed as:

$$
\begin{aligned}
& \mathrm{ESR}=K_{C} \frac{4 \rho l}{\pi N_{a} D_{a}^{2}} \\
& \times\left[1+2\left(\frac{N_{a} D_{a}}{1.155 \sqrt{N_{a}} \times D_{a}+0.0508}\right)^{2}\left(\frac{D_{a} \sqrt{f}}{0.262}\right)^{4}\right] .
\end{aligned}
$$

In the formula, $K_{c}$ is the length correction coefficient, $\rho$ is the resistivity of the copper wire, $l$ is the wire length of the coil, $N_{a}$ is the number of strands of each coil, $D_{a}$ is the diameter of the single-strand coil of the Litz wire, and $f$ is the frequency. Thereby, the internal resistances $R_{1}$ and $R_{2}$ of the primary and secondary coils can be calculated. If the rated power of the system is expressed by $P_{N}$, the output power $P_{\text {out }}$ and the efficiency $\eta$ satisfy:

$$
P_{\text {out }}>P_{N}
$$

$\eta>\eta_{\min }=85 \%$

Based on the above analysis, the coil parameters optimization mathematical model with maximum power factor under the deviation as optimization target and the system parameters as constraints can be described by the following formula:

$$
\left\{\begin{array}{l}
\max (\lambda) \\
\text { s.t. }\left\{\begin{array}{l}
L_{1} \leq \frac{2 \omega_{0} M^{2} U_{C}}{3 U_{1} R_{e}} \\
L_{2} \leq \frac{2 R_{e} U_{C}}{3 \omega_{0} U_{2}} \\
P_{\text {out }}>P_{N} \\
\eta>\eta_{\min }=85 \%
\end{array}\right.
\end{array}\right.
$$

\subsection{Coil parameters design optimization process based on the group method}

Since the coil parameters array satisfying the system is limited, the coil parameters can be optimized by the group method. The specific process is as follows: firstly, the size and mutual inductance of the primary and secondary coils are determined according to the structural parameters of the vehicle body and the input, output requirements of the system. Then determine the number of turns of the primary and secondary coils to verify that the constraints are met. The array that does not satisfy the constraints is culled. The array satisfying the constraints is compared with the previous group. And the array with the smaller self-inductance value is compared with the next group. Finally a set of optimal coil parameters is obtained. Its optimization flow chart is shown in Fig. 7.

\section{Simulation verification}

According to the installation space of the existing non-catenary tram, it is determined that the primary coil adopts a rectangular coil of $20 \mathrm{~m} * 1 \mathrm{~m}$, the secondary coil is a square coil of $1 \mathrm{~m} * 1 \mathrm{~m}$. And the distance between the primary and secondary coils is $10 \mathrm{~cm}$. The single-turn coil model was imported into Ansys Maxwell simulation software. The self-inductance and mutual inductance of the single-turn winding of the structure were obtained as shown in Table 2.

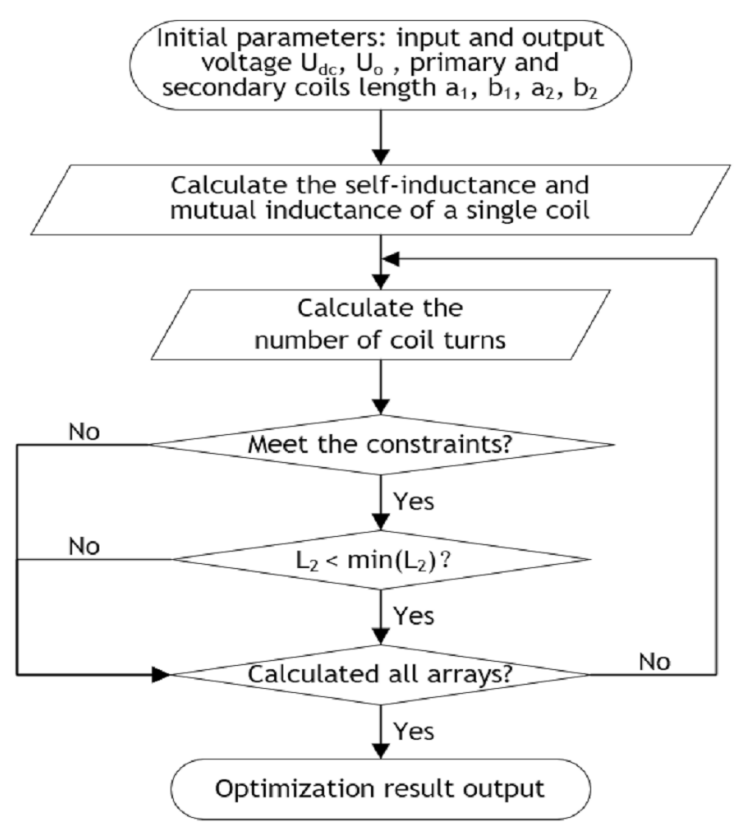

Fig. 7 Flow chart for optimizing coil parameters 
Table 2 Inductance and mutual inductance of single-turn primary and secondary coil

\begin{tabular}{lccc}
\hline Parameters & $L_{10} / \mathrm{uH}$ & $L_{20} / \mathrm{uH}$ & $M / \mathrm{uH}$ \\
\hline Value & 40.20 & 3.64 & 0.85 \\
\hline
\end{tabular}

On this basis, the coil parameters are designed and optimized using the algorithm described in Subsection 4.3. The final results are shown in Table 3 . The system simulation model is built in the software Matlab by using this set of parameters. The simulation results are shown in Fig. 8.

Analysis of the simulation results shows that under this set of parameters, the output power of the system can reach $305 \mathrm{~kW}$. The system efficiency is $95.3 \%$. And the power factor of the system can reach 0.97 , which meets the system requirements.

In order to simulate the differences of coils on the actual trams and verify the reliability of the system output results under different coil precisions, multiple sets of secondary coil parameters are set for simulation. When there are different deviations in the secondary coil self-inductance, the power factor of the system is shown in Table 4. As shown in the table, when the inductance deviation is within $\pm 7 \%$, the system power factor can be maintained above 0.85 , that is, the electromagnetic coupling mechanism has a strong ability to transmit active power, which ensures the normal operation of the system.

\section{Conclusion}

Dynamic charging system for the non-catenary tram puts more stringent requirements on output power and efficiency. Due to factors such as the manufacturing process of coils, unreasonable parameter design will lead to a sharp decrease in the power factor of the system, affecting the normal operation of the vehicle. Based on

Table 3 Theoretical and calculated values of system parameters

\begin{tabular}{lc}
\hline Parameters & Value \\
\hline DC voltage & $U_{d c}=750 \mathrm{~V}$ \\
Frequency & $f=30 \mathrm{kHz}$ \\
Mutual inductance & $M=8.45 \mathrm{uH}$ \\
Turns of primary coil & $N_{1}=2$ turns \\
Turns of secondary coil & $N_{2}=5$ turns \\
Inductance of primary coil & $L_{1}=160.8 \mathrm{uH}$ \\
Inductance of secondary coil & $L_{2}=91.0 \mathrm{uH}$ \\
Resistance of primary coil & $R_{1}=13.10 \mathrm{~m} \Omega$ \\
Resistance of secondary coil & $R_{2}=3.12 \mathrm{~m} \Omega$ \\
Compensation capacitor of primary coil & $C_{1}=176.06 \mathrm{nF}$ \\
Compensation capacitor of secondary coil & $C_{2}=309.55 \mathrm{nF}$ \\
Load & $R e=1.51 \Omega$ \\
\hline
\end{tabular}

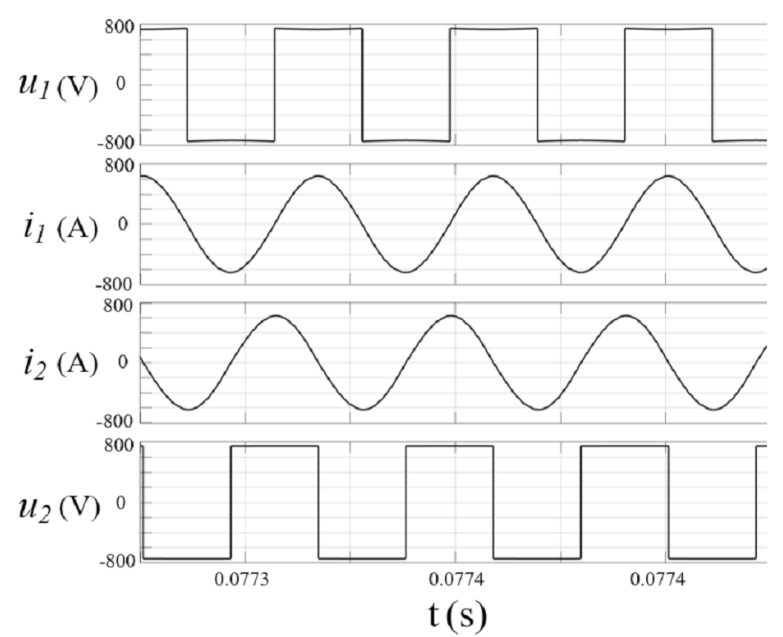

(a)

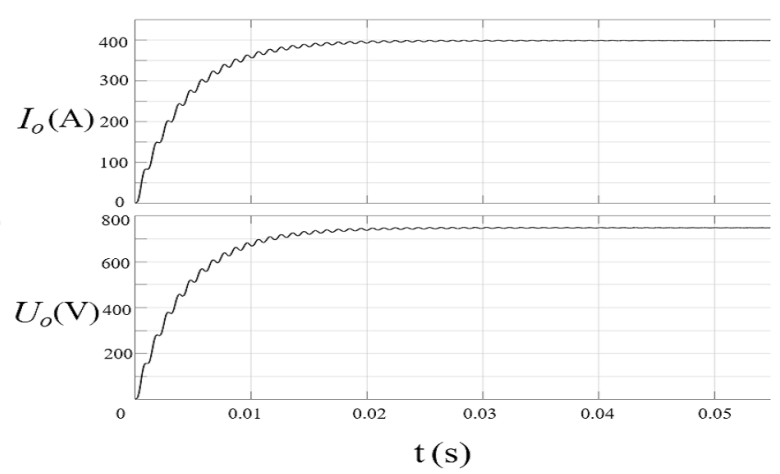

(b)

Fig. 8 Simulation result of Matlab

(a) Primary and secondary voltage and current waveforms of AC side (b) Waveform of output current and voltage

Table 4 The relationship between secondary coil self-inductance and power factor

\begin{tabular}{lccccccc}
\hline$L_{2} / \mathrm{uH}$ & 84 & 86 & 88 & 91 & 93 & 95 & 97 \\
\hline$\lambda$ & 0.85 & 0.88 & 0.94 & 0.97 & 0.94 & 0.89 & 0.85 \\
\hline
\end{tabular}

the engineering practice, this paper proposes a set of parameters design algorithm for inductively coupled coils of the tram. And based on the power factor reduction caused by the differences in self-inductance of the secondary coils, the parameters are optimized from the perspective of hardware design. The simulation results show that the proposed method can effectively select the optimal coil parameter array that meets the system requirements. And under the condition that the system constraints are satisfied, even if the deviation of the secondary coil caused by the coil differences is large, the power factor of the system can be maintained at a high level. The result proves the feasibility and effectiveness of the parameters design method which has high engineering application value. 


\section{Acknowledgement}

The project presented in this article is supported by Fundamental Research Funds for the Central Universities [2018YJS151] and National key research and development program [2017YFB1201003-014].

\section{References}

Budhia, M., Covic, G. A, Boys, J. T. (2011) "Design and Optimization of Circular Magnetic Structures for Lumped Inductive Power Transfer Systems", IEEE Transactions on Power Electronics, 26(11), pp. 3096-3108.

https://doi.org/10.1109/TPEL.2011.2143730

Cai, H., Shi, L., Li, Y. (2014) "Output Power Adjustment in Inductively Coupled Power Transfer System", Transaction of China Electrotechnical Society, 29(01), pp. 215-220. https://doi.org/10.19595/j.cnki.1000-6753.tces.2014.01.030

Fan, M., Shi, L., Yin, Z., Jiang, 1., Zhang, F. (2018) "Research on Control Strategy of Segmented Power Supply Formobile Inductive Power Transfer System", Transaction of China Electrotechnical Society, 33(17), pp. 3998-4006.

https://doi.org/10.19595/j.cnki.1000-6753.tces.171230

Geng, Y., Yang, Z., Lin, F., Wang, J. (2017) "Characteristic Analysis of Multiple-receiving Coupling Coil Mode for Wireless Power Transfer Systems", Transaction of China Electrotechnical Society, 32(S2), pp. 1-9.

https://doi.org/10.19595/j.cnki.1000-6753.tces.170296

Hu, C., Sun, Y., Wang, Z., Tang, C., Xiong, Q. (2013) "Design of magnetic coupler for EVs' wireless charging", International Journal of Applied Electromagnetics and Mechanics, 43(3), pp. 195-205. https://doi.org/10.3233/jae-131693

Li, H. L., Hu, A. P, Covic, G. A. (2010) "A Power Flow Control Method on Primary Side for a CPT System", International Power Electronics Conference (IPEC), pp. 1050-1055. https://doi.org/10.1109/IPEC.2010.5542076

Li, Y., Mai, R., Ma, L., He, Z. (2015) "Dual parallel wound primary coil based IPT systems and its power allocation technique", Proceedings of the CSEE, 35(17), pp. 4454-4460. https://doi.org/10.13334/j.0258-8013.pcsee.2015.17.021

Sun, Y., Xia, C., Dai, X., Su, Y. (2010) "Analysis and optimization of mutual inductance for inductively coupled power transfer system", Proceedings of the CSEE, 30(33), pp. 44-50. https://doi.org/10.13334/j.0258-8013.pcsee.2010.33.007
Su, Yu-G., Zhang, S., Hu, C., Tang, C.-S., Zhou, W. (2016) "An embeddable transmitter coil applied to electric vehicles powered by IPT system", International Journal of Applied Electromagnetics and Mechanics, 50(4), pp. 627-636. https://doi.org/10.3233/JAE-150149

Tan, L., Huang, X., Li, H., Huang, H. (2010) "Study of Wireless Power Transfer System through Strongly Coupled Resonances", In: 2010 International Conference on Electrical and Control Engineering, Wuhan, China, pp. 4275-4278. https://doi.org/10.1109/iCECE.2010.1446

Xu, L., Du, Y., Shi, L. (2018) "Reluctance Circuit Analysis and Structure Optimization of Contactless Transformer", In: 2017 20th International Conference on Electrical Machines and Systems (ICEMS), Sydney, NSW, Australia, pp. 1-4. https://doi.org/10.1109/ICEMS.2017.8055953

Yang, Q., Chen, H., Xu, G., Sun, M., Fu, W. (2010) "Research Progress in Contactless Power Transmission Technology", Transaction of China Electrotechnical Society, 25(07), pp. 6-13. https://doi.org/10.19595/j.cnki.1000-6753.tces.2010.07.002

Zhang, L., Yang, Y., Sun, M., Liu, H. (2017) "Energy Management Strategy Based on Dynamic Programming for Dual Source Trolleybus", Tehnički vjesnik / Technical Gazette, 24(5), pp. 1439-447. https://doi.org/10.17559/TV-20170628043048

Zhao, Z., Liu, F., Chen, K. (2016) "New Progress of Wireless Charging Technology for Electric Vehicles", Transaction of China Electrotechnical Society, 31(20), pp. 30-40. https://doi.org/10.19595/j.cnki.1000-6753.tces.2016.20.003

Zheng, G. (2017) "Optimization Design of Efficient Middle Distance Magnetic-Resonance Wireless Charge Coil Suitable for Electric Vehicle Charging", Transaction of China Electrotechnical Society, 32(S1), pp. 209-216. https://doi.org/10.19595/j.cnki.1000-6753.tces.170302 